\title{
The Neuroprotective Effects of Resveratrol Preconditioning in Transient Global Cerebral Ischemia-Reperfusion in Mice
}

\author{
Shuang LIU, Jinbo SUN, Yanshuang LI \\ Jinan Central Hospital Affiliated to Shandong University, Department of Neurology, Jinan, China
}

\section{ABSTRACT}

AIM: This study was designed to elucidate the neuroprotective effect of resveratrol in a mouse model of bilateral common carotid artery occlusion (BCCAO).

MATERIAL and METHODS: Sixty male C57BL/6 mice, weighing 20-24 g, were used in our experiments. The mice were randomly assigned into three groups: control group, BCCAO group and BCCAO+Resveratrol group. Neurological score was assessed 24h, 48h, 72h after BCCAO, respectively. Hematoxylin and eosin (H\&E) staining, NeuN and TUNEL were performed to detect the neuronal death and survival. The expression of Bcl-2, Bax, caspase-3, and cleaved caspase-3 were also detected to assess the anti-apoptotic effect of resveratrol by Western Blot.

RESULTS: Resveratrol significantly improved neurological score in BCCAO mice. Besides, it attenuates neuronal apoptosis via increasing the expression of $\mathrm{Bcl}-2$ and decreasing the expression of Bax, caspase-3, and cleaved caspase- 3 . Resveratrol promotes neuronal survival in mice subjected to BCCAO.

CONCLUSION: Resveratrol is beneficial in the model of BCCAO, which is associated with its anti-apoptotic effect.

KEYWORDS: Resveratrol, Apoptosis, Global cerebral ischemia

\section{INTRODUCTION}

Ischemic stroke is caused by a transient or permanent occlusion in cerebral vessels. It causes neuronal death and associated behavioral deficits, including sensorimotor dysfunction, spatial orientation disorder, and learning and memory impairments $(1,13,22)$. Stroke may induce a series of neurological disorders, such as oxidative stress, blood-brain barrier dysfunction, neuronal apoptosis and inflammation (8).

Resveratrol is a polyphenol found in high amounts in grapes, berries, peanuts, and medicinal plants such as Polygonum cuspidatum (2). It is produced by plants to protect themselves against stress, excessive sunlight, ultraviolet radiation, inflammation, and fungi (27). It was reported to be the solution to the "French Paradox", a term used to describe the observa- tion that the French population had a very low incidence of cardiovascular disease despite a high consumption of wine and saturated fat (17). Recently, resveratrol has received widespread attention for its potential use as a therapeutic agent in the prevention and treatment of numerous diseases, such as hypertension (10), coronary artery disease (21) and diabetes (30). Previous studies have suggested that resveratrol exhibits anti-inflammatory, anti-oxidant, and anti-carcinogenic properties $(3,6,14,28,33)$.

This study aims to investigate whether resveratrol had a neuroprotective effect on ischemic neurons in a bilateral common carotid artery occlusion model. If so, we also wanted to find out whether it exhibited anti-apoptotic effect in this ischemic model. 


\section{MATERIAL and METHODS}

\section{Animals}

Sixty C57BL/6 mice, weighing 20-24 g, were used in our experiments and they were provided by the Experimental Animal Center of the Shandong University. Mice were kept in a controlled-temperature room with a $12 \mathrm{~h} / 12 \mathrm{~h}$ light/dark cycle. All the mice were allowed free access to food and tap water.

\section{Establishment of Global Cerebral Ischemia}

Bilateral common carotid artery occlusion (BCCAO) was used to trigger global cerebral ischemia. Mice were anesthetized with $3 \%$ isoflurane. After induction, the isoflurane concentration was maintained at $1.5 \%$. A midline incision was made between the neck and sternum and the trachea was exposed. Then both the right and left common carotid arteries were carefully separated. Cerebral ischemia was induced by clamping both of the arteries with two miniature artery clips. After 20 minutes of cerebral ischemia, the clips were removed to allow for the reperfusion of blood through the carotid arteries. Sham-operated mice underwent the same surgical procedure without artery occlusion. During the surgery, the body temperature was monitored with a temperature probe and maintained at $37.0-37.5^{\circ} \mathrm{C}$ using a heating pad.

\section{Drug Administration}

For the $\mathrm{BCCAO}+$ Resveratrol group, Resveratrol (dissolved in $2 \%$ dimethyl sulfoxide (DMSO)) was administered at a dose of $30 \mathrm{mg} / \mathrm{kg}$ i.p. for 7 consecutive days before surgery. Mice in the sham group and BCCAO group were injected solely with an equal concentration and amount of DMSO.

\section{Neurological Tests}

The treated mice were left to recover for $24 \mathrm{~h}$ before subsequent tests. The motor deficits of mice were tested with some modifications. Briefly, mice were placed on a 10 - to $20-\mathrm{cm}$ screen (grid size $0.2 \times 0.2 \mathrm{~cm}$ ) that could rotate from $0^{\circ}$ (horizontal) to $90^{\circ}$ (vertical). Mice were placed on the screen, which was in a horizontal position at first, and the screen was then rotated into the vertical position. The duration of each mouse on the vertical screen was recorded up to a maximum of $15 \mathrm{~s}$ (corresponding to a maximum of three points). Subsequently, each mouse was placed at the center of a horizontal wooden rod $(1.5 \mathrm{~cm}$ in diameter), and the duration that the mouse was able to keep balanced on the rod was recorded up to a maximum of 30 s (corresponding to a maximum of three points). Finally, a prehensile traction test was performed. The time that the mouse was able to cling to a horizontal rope was recorded up to a maximum of $5 \mathrm{~s}$ (corresponding to a maximum of three points). A total motor score (9 possible points) was recorded. The neurologic tests were performed at 24-, 48-, and 72-h post-reperfusion by an observer who was blind to the grouping. The total motor score (TMS) has been shown to be an accurate test for evaluating global cerebral ischemia injury in mice $(11,26)$.

\section{Hematoxylin and Eosin (H\&E) Staining}

Neuronal damage was assessed using H\&E staining. Three days after the induction of ischemia, animals were anesthetized with sodium pentobarbital (50 mg/kg i.p.) and transcardially perfused with $4 \%$ phosphate-buffered paraformaldehyde after a flush with $0.1 \mathrm{M}$ phosphate-buffered saline. Brains were removed, and post-fixed at $4^{\circ} \mathrm{C}$ in $4 \%$ paraformaldehyde overnight and then cut in a freezing microtome. Six consecutive sections $(12 \mu \mathrm{m})$ taken backward from the optic chiasm and that included the dorsal hippocampus were stained with H\&E. The pyramidal neurons of the CA1 region were evaluated.

\section{NeuN and TUNEL Analysis}

The sections were permeabilized with $0.4 \%$ Triton X-100-PBS for $10 \mathrm{~min}$, and were then incubated with $10 \%$ normal donkey serum for $2 \mathrm{~h}$ at room temperature. The sections were next incubated with anti-NeuN antibody (1:1000, mouse) overnight at $4^{\circ} \mathrm{C}$, followed by three times wash with PBS. Then the sections were incubated with the secondary antibody (Alexa Fluor 594 donkey anti-mouse lgG) at room temperature for 2 hours, followed by three times wash in PBS. For TUNEL staining, sections were incubated with TUNEL reaction mixture in a dark humidified chamber for $1 \mathrm{~h}$ at $37^{\circ} \mathrm{C}$, followed by a final wash for $3 \times 10 \mathrm{~min}$ with PBS. The sections were viewed and analyzed using laser scanning confocal microscopy (LSCM, Olympus FV1000).

\section{Western Blot}

Protein content was determined with the bicinchoninic acid (BCA) protein assay, and protein samples were separated by electrophoresis on sodium dodecyl sulfate-polyacrylamide gel (SDS-PAGE) and transferred to a polyvinylidene difluoride membrane. The membranes were then blocked with 5\% fat-free milk and incubated overnight with the appropriate primary antibodies, respectively [anti-Bcl-2 (1:200), anti-Bax (1:200), anti- $\beta$-actin (1:5000) (SantaCruz Biotechnology), anti-caspase-3 (1:1000), anti-cleaved caspase3 (1:1000) (Cell Signalling Technology, Beverly, MA, USA)]. After being extensively washed with Tris-buffered saline containing $0.1 \%$ Tween 20 buffer, the membranes were incubated with secondary antibodies (Santa Cruz Inc, Santa Cruz, CA; $1: 2000)$ for 2 hours at room temperature, and washed with Tris-buffered saline, $0.1 \%$ Tween 20 (TBST) for three times. The protein bands were detected using a Bio-Rad imaging system (Bio-Rad, Hercules, CA, USA) and quantified using the Quantity One software package (West Berkeley, CA, USA).

\section{Statistical Analysis}

The statistical analyses were conducted using SPSS 11.0 for Windows software (SPSS Inc., Chicago, IL). All values, except for total motor scores, are presented as the means \pm the SDs and were analyzed using a one-way analysis of variance. Between-group differences were detected based on post hoc Student-Newman-Keuls tests. The total motor scores are expressed as the medians and were analyzed using the Kruskal-Wallis test. Values of $P<0.05$ were considered to be statistically significant. 


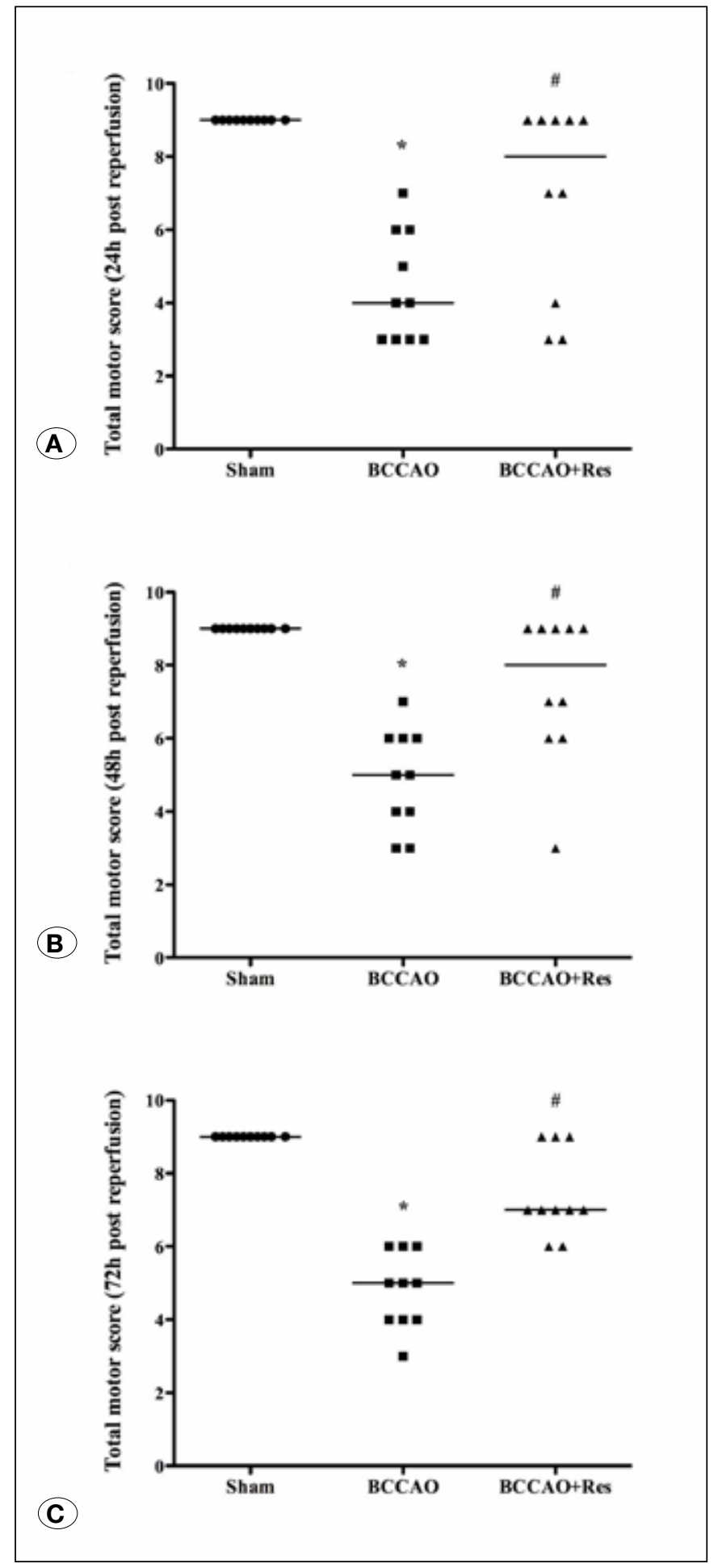

Figure 1: Neurologic scores of each animal at 24 (A), 48 (B), and 72 (C) hours after reperfusion. Significantly, the total motor scores (TMS) in the BCCAO+Resveratrol group were significantly better than those in the BCCAO groups at 24, 48, and 72 hours after reperfusion ( $n=10,{ }^{*} p<0.05$ vs. Sham group, ${ }^{*} p<0.05$ vs. BCCAO group).

\section{RESULTS}

\section{Neurological Score}

In the Figure $1 \mathrm{~A}-\mathrm{C}$, the neurological score in BCCAO group decreased dramatically compared with that in sham group $(p<0.05)$ at $24 \mathrm{~h}, 48 \mathrm{~h}$ and $72 \mathrm{~h}$ after the induction of ischemia. Resveratrol pre-treatment ameliorated the injury and the neurological score was elevated.

\section{H\&E Staining}

Three days after reperfusion, the number of viable neurons in the CA1 region was dramatically decreased. Resveratrol pretreatment significantly reduced the neuronal degeneration in the $\mathrm{CA} 1$ region compared with that in the sham group (Figure $2 A-D)$.

\section{NeuN and TUNEL}

As shown in the Figure 3, ischemia triggered a significant loss of neurons in the CA1 region as indicated by the decrease of NeuN-positive cells compared with the sham group. However, the survival of neurons was dramatically increased when resveratrol was given. Besides, ischemia induced a dramatic neuronal apoptosis in comparison with the sham group.

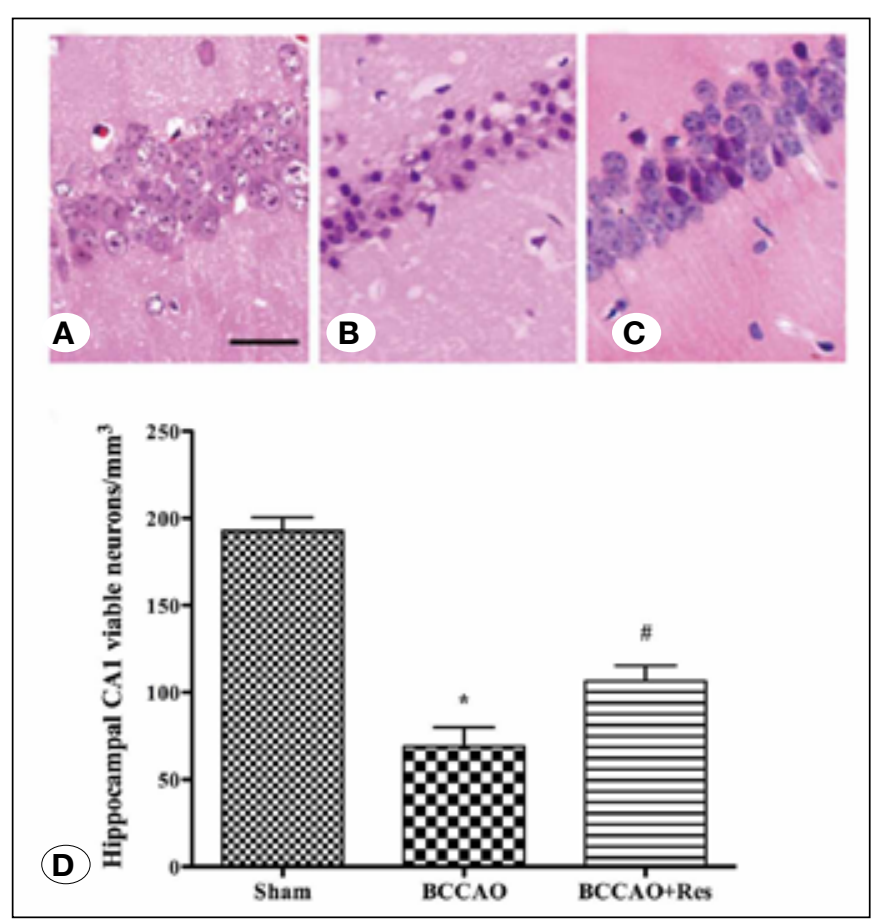

Figure 2: Resveratrol preconditioning protects hippocampal CA1 neurons against ischemic injury $(\mathbf{A}, \mathbf{B}, \mathbf{C})$. Representative microphotographs of H\&E-stained neurons in hippocampal CA1 regions at 72 hours after reperfusion in mice. (A) Sham, (B) BCCAO 72 hours, (C) BCCAO+Resveratrol 72 hours. (D) Viable CA1 neurons were counted and analyzed at $72 \mathrm{~h}$ after reperfusion. Viable neurons were significantly decreased in the CA1 region in BCCAO group compared with those in the sham group. While viable neurons were dramatically increased the BCCAO+Resveratrol group compared with those in BCCAO group at 72 hours after reperfusion ( $\mathrm{n}=10,{ }^{*} p<0.05$ vs. Sham group, ${ }^{*} p<0.05$ vs. BCCAO group). 
However, resveratrol pre-treatment attenuated the apoptosis of neurons as indicated by the decrease of TUNEL-positive neurons in the CA1 region.

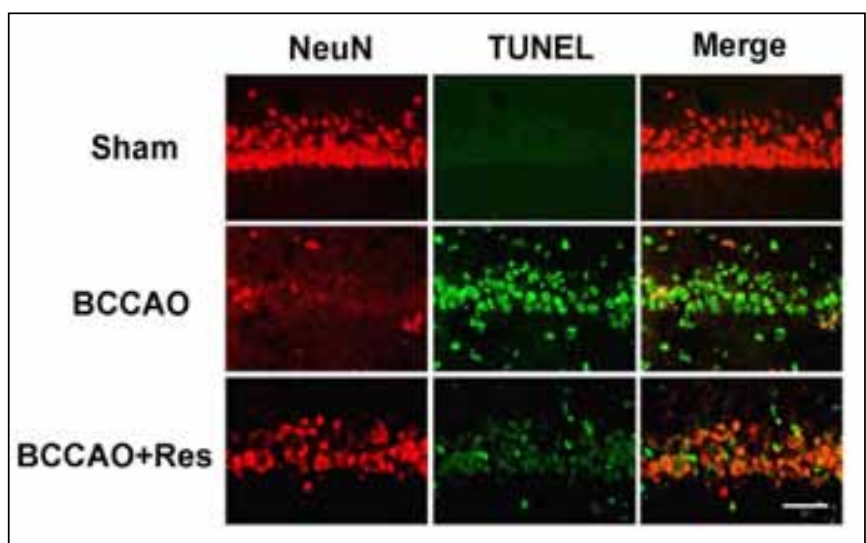

Figure 3: Resveratrol preconditioning significantly attenuates neuronal cell death in the hippocampal CA1 region. Representative hippocampal CA1 from sham, BCCAO, BCCAO+Resveratrol groups were subjected to immunofluorescence labeling with NeuN (Red) and TUNEL (Green) staining. The results suggest that neuronal apoptosis was increased dramatically in BCCAO group compared with that in sham group. While resveratrol preconditioning significantly attenuated neuronal apoptosis compared with that in BCCAO group. Scale bar=50 $\mathrm{mm}(\mathrm{n}=10)$.

\section{Western Blot}

The expression of the anti-apoptotic protein $\mathrm{Bcl}-2$ and the pro-apoptotic protein Bax was displayed in Figure 4 $\mathrm{A}, \mathrm{B}$. BCCAO lowered Bcl-2 expression and increased Bax expression in comparison with the sham group. On the contrary, in comparison with BCCAO group, pre-treatment with resveratrol elevated $\mathrm{Bcl}-2$ expression and decreased Bax expression. In addition, in comparison with the sham group, BCCAO-induced caspase- 3 and cleaved caspase- 3 up-regulation was markedly abolished by resveratrol pretreatment (Figure 4 C,D).

\section{DISCUSSION}

The results of this study demonstrate that resveratrol preconditioning has a neuroprotective effect in cerebral ischemiareperfusion in mice. Resveratrol preconditioning significantly improved the TMS and reduced neuronal death after cerebral ischemia-reperfusion. We found that resveratrol preconditioning induced an anti-apoptotic effect, which is shown by its ability to increase $\mathrm{Bcl}-2$ expression and to decrease the expression of Bax, cleaved caspase-3 and caspase-3.

Pyramidal neurons of the CA1 region are particularly vulnerable to ischemic injury. This region undergoes delayed neuronal death, often reported as apoptosis united with DNA fragmentation (20). It has been suggested that resveratrol can cross the blood-brain barrier and protect against global

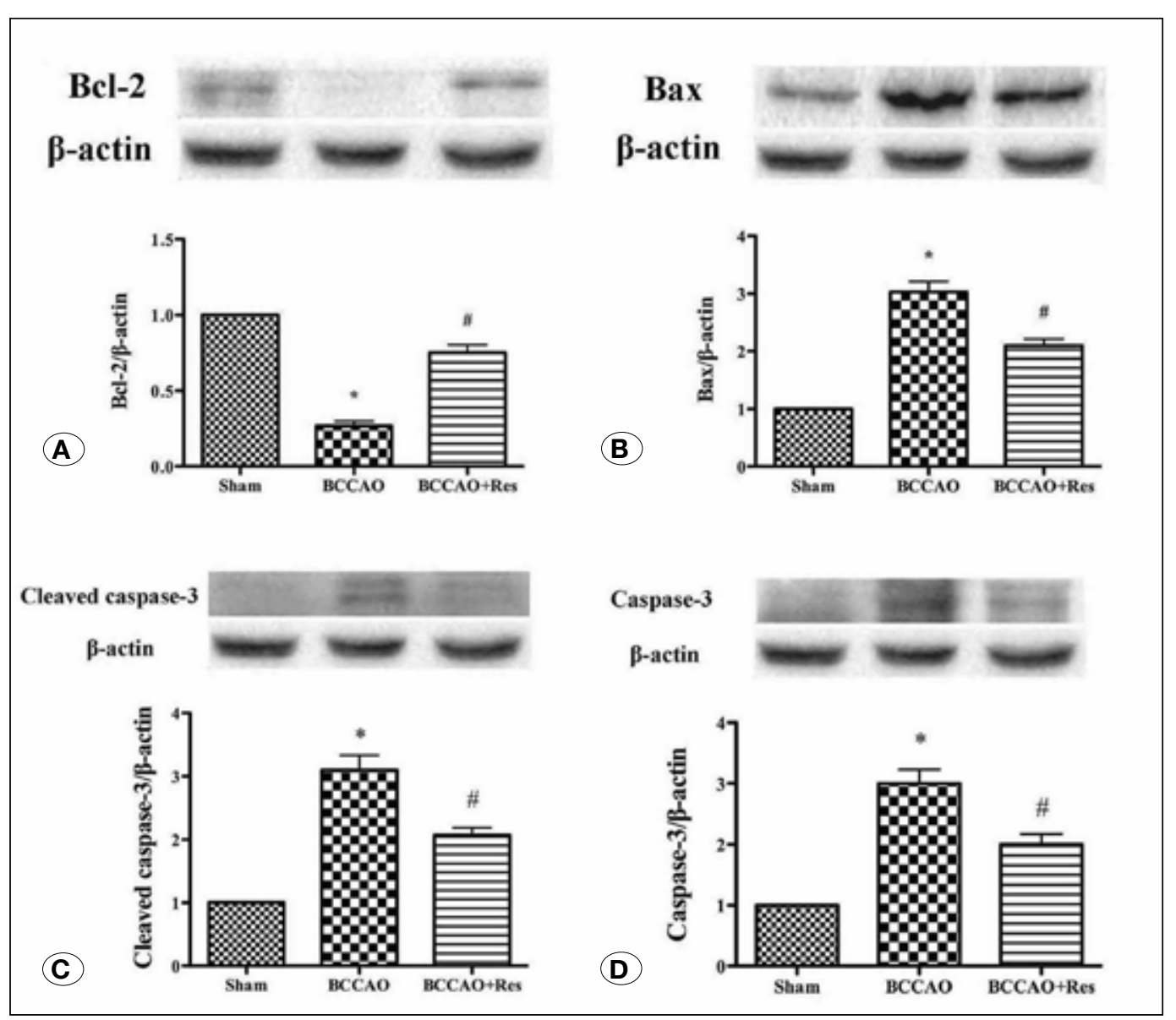

Figure 4: The expressions of $\mathrm{Bcl}-2$ (A), Bax (B), cleaved caspase-3 (C), and caspase-3 (D) detected by Western blot in each group. Ischemia downregulated $\mathrm{Bcl}-2$ expression dramatically and upregulated the expression of Bax, cleaved caspase-3 and caspase- 3 significantly. Resveratrol preconditioning increased $\mathrm{Bcl}-2$ expression dramatically and decreased the expression of Bax, cleaved caspase- 3 and caspase-3 markedly $(\mathrm{n}=10$, $" p<0.05$ vs. Sham group, $" p$ $<0.05$ vs. BCCAO group). 
cerebral ischemic injury and inhibit glial cell activation (32). In a rat model of permanent bilateral common carotid artery occlusion, resveratrol improved learning and memory abilities, which are associated with its anti-oxidant effects (18). Nitric oxide was reported to play a critical role in the neuroprotective effects of resveratrol in focal cerebral ischemia $(12,28)$. In addition, resveratrol protects rat brain from cerebral ischemic injury via a sirtuin 1-uncoupling protein 2 and TRPC6/CREB pathways $(7,16)$. Resveratrol also regulates the expression of various proteins associated with oxidative stress and energy metabolism in focal cerebral ischemia (25). Furthermore, resveratrol can enhance the neuroprotection when coadministrated with lipoic acid in cerebral ischemia (24). In the present study, we first established the model of BCCAO, and then we found that resveratrol pretreatment could protect the brain from ischemic injury, which is associated with its antiapoptotic effect.

Neuronal apoptosis is a key pathological process of ischemic stroke $(4,9)$. Several experimental studies have demonstrated that the inhibition of apoptosis reduces ischemic injury (23). Though two pathways of apoptosis (the extrinsic and the intrinsic) have been demonstrated (5), the final phase of apoptosis execution that includes activation of executioner caspases (e.g., caspase 3) is shared by both these pathways (29). Interactions between the pro-apoptotic Bax and antiapoptotic Bcl-2 family proteins on the mitochondria are believed to play an important role in cell survival (31). The antiapoptotic protein $\mathrm{Bcl}-2$ is capable of preventing cytochrome $\mathrm{c}$ release, which is an activator of apoptosis, and promotes cell survival, while the pro-apoptotic protein (Bax) promotes cell death (19). Resveratrol has been reported to up-regulate Bcl-2 expression in a rat model of focal cerebral ischemia (15). So in the present study, we detected the expression of Bax and Bcl2 using Western blot, and the results suggest that resveratrol increases $\mathrm{Bcl}-2$ expression and decreases Bax expression, indicating that the protective effect against cerebral ischemia is related to inhibiting apoptosis. Moreover, we also assayed the expression of caspase- 3 and cleaved caspase-3, and the results suggest that resveratrol pretreatment lowers the expression of the two proteins, indicating that resveratrol protects the brain from ischemia via inhibiting the final phase of apoptosis.

\section{CONCLUSION}

Resveratrol provides significant neuroprotection in mice subjected to global cerebral ischemia via inhibiting neuronal apoptosis (increasing $\mathrm{Bcl}-2$ expression and decreasing the expressions of Bax, caspase-3 and cleaved caspase-3).

\section{- REFERENCES}

1. Amano M, Hasegawa M, Hasegawa T, Nabeshima T: Characteristics of transient cerebral ischemia-induced deficits on various learning and memory tasks in male Mongolian gerbils. Jpn J Pharmacol 63: 469-477, 1993

2. Baur JA, Sinclair DA: Therapeutic potential of resveratrol: The in vivo evidence. Nat Rev Drug Discov 5: 493-506, 2006
3. Brito PM, Simoes NF, Almeida LM, Dinis TC: Resveratrol disrupts peroxynitrite-triggered mitochondrial apoptotic pathway: A role for Bcl-2. Apoptosis 13: 1043-1053, 2008

4. Brouns R, De Deyn PP: The complexity of neurobiological processes in acute ischemic stroke. Clin Neurol Neurosurg 111: 483-495, 2009

5. Chen M, Wang J: Initiator caspases in apoptosis signaling pathways. Apoptosis 7: 313-319, 2002

6. Csiszar A: Anti-inflammatory effects of resveratrol: Possible role in prevention of age-related cardiovascular disease. Ann N Y Acad Sci 1215: 117-122, 2011

7. Della-Morte D, Dave KR, DeFazio RA, Bao YC, Raval AP, Perez-Pinzon MA: Resveratrol pretreatment protects rat brain from cerebral ischemic damage via a sirtuin 1-uncoupling protein 2 pathway. Neuroscience 159: 993-1002, 2009

8. Diaz-Ruiz A, Zavala C, Montes S, Ortiz-Plata A, SalgadoCeballos H, Orozco-Suarez S, Nava-Ruiz C, Perez-Neri I, Perez-Severiano F, Rios C: Antioxidant, antiinflammatory and antiapoptotic effects of dapsone in a model of brain ischemia/ reperfusion in rats. J Neurosci Res 86: 3410-3419, 2008

9. Doyle KP, Simon RP, Stenzel-Poore MP: Mechanisms of ischemic brain damage. Neuropharmacology 55: 310-318, 2008

10. Franco JG, Lisboa PC, Lima NS, Amaral TA, Peixoto-Silva N, Resende AC, Oliveira E, Passos MC, Moura EG: Resveratrol attenuates oxidative stress and prevents steatosis and hypertension in obese rats programmed by early weaning. $J$ Nutr Biochem 24: 960-966, 2013

11. Homi HM, Mixco JM, Sheng H, Grocott HP, Pearlstein RD, Warner DS: Severe hypotension is not essential for isoflurane neuroprotection against forebrain ischemia in mice. Anesthesiology 99: 1145-1151, 2003

12. Huang SS, Tsai MC, Chih CL, Hung LM, Tsai SK: Resveratrol reduction of infarct size in Long-Evans rats subjected to focal cerebral ischemia. Life Sci 69: 1057-1065, 2001

13. Jung JE, Kim GS, Chen H, Maier CM, Narasimhan P, Song YS, Niizuma K, Katsu M, Okami N, Yoshioka H, Sakata H, Goeders $\mathrm{CE}$, Chan PH: Reperfusion and neurovascular dysfunction in stroke: From basic mechanisms to potential strategies for neuroprotection. Mol Neurobiol 41: 172-179, 2010

14. Lanzilli G, Cottarelli A, Nicotera G, Guida S, Ravagnan G, Fuggetta MP: Anti-inflammatory effect of resveratrol and polydatin by in vitro IL-17 modulation. Inflammation 35: 240248, 2012

15. Li Z, Pang L, Fang F, Zhang G, Zhang J, Xie M, Wang L: Resveratrol attenuates brain damage in a rat model of focal cerebral ischemia via up-regulation of hippocampal Bcl-2. Brain Res 1450:116-124, 2012

16. Lin Y, Chen F, Zhang J, Wang T, Wei X, Wu J, Feng Y, Dai Z, Wu Q: Neuroprotective effect of resveratrol on ischemia/ reperfusion injury in rats through TRPC6/CREB pathways. J Mol Neurosci 50: 504-513, 2013

17. Liu BL, Zhang X, Zhang W, Zhen HN: New enlightenment of French Paradox: Resveratrol's potential for cancer chemoprevention and anti-cancer therapy. Cancer Biol Ther 6:1833-1836, 2007 
18. Ma X, Sun Z, Liu Y, Jia Y, Zhang B, Zhang J: Resveratrol improves cognition and reduces oxidative stress in rats with vascular dementia. Neural Regen Res 8: 2050-2059, 2013

19. Martinou JC, Youle RJ: Mitochondria in apoptosis: Bcl-2 family members and mitochondrial dynamics. Dev Cell 21 : 92-101, 2011

20. Miyamoto O, Tamae K, Kasai H, Hirakawa H, Hayashida $\mathrm{Y}$, Konishi R, Itano T: Suppression of hyperemia and DNA oxidation by indomethacin in cerebral ischemia. Eur $\mathrm{J}$ Pharmacol 459:179-186, 2003

21. Movahed A, Yu L, Thandapilly SJ, Louis XL, Netticadan T: Resveratrol protects adult cardiomyocytes against oxidative stress mediated cell injury. Arch Biochem Biophys 527:74-80, 2012

22. Murray V, Norrving B, Sandercock PA, Terent A, Wardlaw JM, Wester P: The molecular basis of thrombolysis and its clinical application in stroke. J Intern Med 267:191-208, 2010

23. Rabuffetti M, Sciorati C, Tarozzo G, Clementi E, Manfredi AA, Beltramo M: Inhibition of caspase-1-like activity by AcTyr-Val-Ala-Asp-chloromethyl ketone induces long-lasting neuroprotection in cerebral ischemia through apoptosis reduction and decrease of proinflammatory cytokines. $J$ Neurosci 20:4398-4404, 2000

24. Saleh MC, Connell BJ, Rajagopal D, Khan BV, Abd-El-Aziz AS, Kucukkaya I, Saleh TM: Co-administration of resveratrol and lipoic acid, or their synthetic combination, enhances neuroprotection in a rat model of ischemia/reperfusion. PLoS One 9: e87865, 2014

25. Shah FA, Gim SA, Kim MO, Koh PO: Proteomic identification of proteins differentially expressed in response to resveratrol treatment in middle cerebral artery occlusion stroke model. J Vet Med Sci 76: 1367-1374, 2014

26. Sheng H, Laskowitz DT, Mackensen GB, Kudo M, Pearlstein $\mathrm{RD}$, Warner DS: Apolipoprotein E deficiency worsens outcome from global cerebral ischemia in the mouse. Stroke 30:11181124, 1999
27. Singh CK, Kumar A, Lavoie HA, Dipette DJ, Singh US: Diabetic complications in pregnancy: Is resveratrol a solution? Exp Biol Med (Maywood) 238: 482-490, 2013

28. Subramanian M, Balasubramanian P, Garver H, Northcott C, Zhao H, Haywood JR, Fink GD, Mohan Kumar SM, Mohan Kumar PS: Chronic estradiol-17 beta exposure increases superoxide production in the rostral ventrolateral medulla and causes hypertension: Reversal by resveratrol. Am J Physiol Regul Integr Comp Physiol 300: R1560-1568, 2011

29. Thornberry NA, Lazebnik Y: Caspases: Enemies within. Science 281:1312-1316, 1998

28. Tsai SK, Hung LM, Fu YT, Cheng H, Nien MW, Liu HY, Zhang FB, Huang SS: Resveratrol neuroprotective effects during focal cerebral ischemia injury via nitric oxide mechanism in rats. J Vasc Surg 46: 346-353, 2007

30. Vecchione C, Aretini A, Marino G, Bettarini U, Poulet R, Maffei A, Sbroggio M, Pastore L, Gentile MT, Notte A, lorio L, Hirsch E, Tarone G, Lembo G: Selective Rac-1 inhibition protects from diabetes-induced vascular injury. Circ Res 98: 218-225, 2006

31. Vela L, Gonzalo O, Naval J, Marzo I: Direct interaction of $\mathrm{Bax}$ and Bak proteins with $\mathrm{Bcl}-2$ homology domain 3 (BH3)-only proteins in living cells revealed by fluorescence complementation. J Biol Chem 288:4935-4946, 2013

32. Wang Q, Xu J, Rottinghaus GE, Simonyi A, Lubahn D, Sun GY, Sun AY: Resveratrol protects against global cerebral ischemic injury in gerbils. Brain Res 958:439-447, 2002

33. Wong JC, Fiscus RR: Resveratrol at anti-angiogenesis/ anticancer concentrations suppresses protein Kinase $G$ signaling and decreases IAPs expression in HUVECs. Anticancer Res 35:273-281, 2015 